\title{
Natural history of colloid cysts of the third ventricle
}

\author{
Thomas L. Beaumont, MD, PhD, ${ }^{1}$ David D. Limbrick Jr., MD, PhD, ${ }^{1}$ Keith M. Rich, MD, ${ }^{1}$ \\ Franz J. Wippold II, MD, ${ }^{2}$ and Ralph G. Dacey Jr., MD ${ }^{1}$
}

1Department of Neurological Surgery and 'Mallinckrodt Institute of Radiology, Washington University School of Medicine, St. Louis, Missouri

\begin{abstract}
OBJECTIVE Colloid cysts are rare, histologically benign lesions that may result in obstructive hydrocephalus and death. Understanding the natural history of colloid cysts has been challenging given their low incidence and the small number of cases in most reported series. This has complicated efforts to establish reliable prognostic factors and surgical indications, particularly for asymptomatic patients with incidental lesions. Risk factors for obstructive hydrocephalus in the setting of colloid cysts remain poorly defined, and there are no grading scales on which to develop standard management strategies.

METHODS The authors performed a single-center retrospective review of all cases of colloid cysts of the third ventricle treated over nearly 2 decades at Washington University. Univariate analysis was used to identify clinical, imaging, and anatomical factors associated with 2 outcome variables: symptomatic clinical status and presentation with obstructive hydrocephalus. A risk-prediction model was defined using bootstrapped logistic regression. Predictive factors were then combined into a simple 5-point clinical scale referred to as the Colloid Cyst Risk Score (CCRS), and this was evaluated with receiver-operator characteristics.
\end{abstract}

RESULTS The study included 163 colloid cysts, more than half of which were discovered incidentally. More than half of the incidental cysts (58\%) were followed with surveillance neuroimaging (mean follow-up 5.1 years). Five patients with incidental cysts (8.8\%) progressed and underwent resection. No patient with an incidental, asymptomatic colloid cyst experienced acute obstructive hydrocephalus or sudden neurological deterioration in the absence of antecedent trauma. Nearly half (46.2\%) of symptomatic patients presented with hydrocephalus. Eight patients (12.3\%) presented acutely, and there were 2 deaths due to obstructive hydrocephalus and herniation. The authors identified several factors that were strongly correlated with the 2 outcome variables and defined third ventricle risk zones where colloid cysts can cause obstructive hydrocephalus. No patient with a lesion outside these risk zones presented with obstructive hydrocephalus. The CCRS had significant predictive capacity for symptomatic clinical status (area under the curve [AUC] 0.917) and obstructive hydrocephalus (AUC 0.845). A CCRS $\geq 4$ was significantly associated with obstructive hydrocephalus $(p<$ 0.0001, RR 19.4).

CONCLUSIONS Patients with incidentally discovered colloid cysts can experience both lesion enlargement and symptom progression or less commonly, contraction and symptom regression. Incidental lesions rarely cause acute obstructive hydrocephalus or sudden neurological deterioration in the absence of antecedent trauma. Nearly one-half of patients with symptomatic colloid cysts present with obstructive hydrocephalus, which has an associated $3.1 \%$ risk of death. The CCRS is a simple 5-point clinical tool that can be used to identify symptomatic lesions and stratify the risk of obstructive hydrocephalus. External validation of the CCRS will be necessary before objective surgical indications can be established. Surgical intervention should be considered for all patients with CCRS $\geq 4$, as they represent the high-risk subgroup.

http://thejns.org/doi/abs/10.3171/2015.11.JNS151396

KEY WORDS colloid cyst; third ventricle; hydrocephalus; oncology

$\mathrm{C}$ OLLOID cysts of the third ventricle are rare, histologically benign lesions that occur in the region of the foramen of Monro, where they may cause obstructive hydrocephalus, rapid clinical deterioration, and death.
The incidence of this lesion has been estimated at 3.2 per million per year, accounting for approximately $2 \%$ of all intracranial tumors. ${ }^{8}$ Reliably defining the natural history of colloid cysts has been challenging due to their low in- 
cidence and the small number of cases in most reported series. Based on previous studies, over $50 \%$ of patients with colloid cysts of the third ventricle are symptomatic at the time of diagnosis and ultimately undergo neurosurgical intervention..$^{3,10,13,14}$ Most of these patients present with symptoms of hydrocephalus, including headache, nausea, vomiting, blurred vision, gait ataxia, and cognitive decline. ${ }^{4,5,8,13,14}$ Notably, a small proportion of symptomatic patients present with acute obstructive hydrocephalus and progress to death despite rapid CSF diversion and appropriate supportive care. Estimates of the risk of rapid clinical deterioration due to acute hydrocephalus in patients with colloid cysts vary widely, from $3 \%$ to $35 \%$, with an associated $5 \%-38 \%$ risk of death..$^{2,4,12,13,17}$ Historically, these patients were often described as having experienced "sudden deterioration and death," but several more recent studies using modern neuroimaging techniques have demonstrated that nearly all patients with acute obstructive hydrocephalus have progressive symptoms preceding precipitous clinical deterioration. Potential exceptions are the rare instances of acute intralesional hemorrhage or "cyst apoplexy.", 10

Incomplete understanding of the natural history of colloid cysts of the third ventricle has impeded efforts to establish evidence-based prognostic factors and surgical indications, particularly for asymptomatic patients with incidentally discovered lesions who are often lost to follow-up. Early studies have focused on patient demographic characteristics, presenting symptoms, and cyst diameter in an attempt to identify patients at risk for rapid deterioration and death. While no pathognomonic findings were identified, patients with colloid cysts of the third ventricle who experience rapid deterioration and death tend to be younger, to have long-standing symptoms, and to have cysts at least $8 \mathrm{~mm}$ in diameter. ${ }^{2,3,17}$ As a result, asymptomatic patients with lesions less than $7 \mathrm{~mm}$ have historically undergone observation with serial imaging, while those with larger lesions have undergone cyst resection or CSF shunting. ${ }^{11}$ With widespread use of modern neuroimaging, an increasing number of colloid cysts are being identified incidentally. Thus, new emphasis must be placed on understanding the natural history of asymptomatic lesions and identifying risk factors associated with symptomatic colloid cysts. Two consecutive studies from the Mayo Clinic have provided several key insights. Importantly, the 10-year incidence of cyst-related symptoms in asymptomatic patients undergoing follow-up with serial imaging was $8 \%$, with only 2 patients demonstrating cyst enlargement, one of whom also developed progressive ventriculomegaly and underwent resection of the lesion. ${ }^{15}$ Symptomatic patients were significantly younger (mean 44 vs 57 years), were more likely to have headaches (78\% vs 28\%), had larger cysts (mean $13 \mathrm{~mm}$ vs $8 \mathrm{~mm}$ ), and were more likely to have ventriculomegaly $(83 \%$ vs $31 \%$ ) and signal hyperintensity on T2-weighted MRI (44\% vs $8 \%)^{14,15}$ Although several surgical case series corroborate these findings, objective prognostic criteria for patients with colloid cysts remain undefined, leaving surgical decision making to individual clinician judgment. ${ }^{1,9,18}$

Here we report on a cohort of all patients evaluated for colloid cysts of the third ventricle at a large tertiary care hospital over nearly 2 decades. Specific emphasis was placed on natural history, presenting symptoms, neuroimaging findings, and morphological characteristics that could be used as potential prognostic factors for symptomatic colloid cysts and the development of obstructive hydrocephalus. With this approach, we developed a simple clinical tool, referred to as the Colloid Cyst Risk Score (CCRS), to aid in clinical decision making and provide a foundation on which objective surgical indications could be established.

\section{Methods \\ Patient Population}

We performed a retrospective review of all cases of colloid cyst of the third ventricle managed at Barnes-Jewish Hospital/Washington University Department of Neurological Surgery between 1996 and 2014. The study was performed with the approval of the Washington University Institutional Review Board in accordance with the Health Insurance Portability and Accountability Act (HIPAA). Cases were identified using the Mallinckrodt Institute of Radiology imaging database and the Neurological Surgery coding database. In the former, broad initial searches using "colloid cyst" were performed and all studies pertaining to regions other than the brain (i.e., thyroid) were discarded. The coding database search was performed for the International Classification of Diseases, Ninth Revision (ICD-9) diagnosis codes 225 and 331.4, indicating "benign neoplasm of brain" and "obstructive hydrocephalus," respectively. The selected records were queried for "colloid cyst" and the resulting list was subsequently combined with the imaging database search results. A final list of nonredundant cases was generated, and neuroimaging and pathology data were reviewed to confirm the presence of a colloid cyst. Cases were excluded if detailed clinical information was unavailable, the lesion was questionably present on neuroimaging, the pathological diagnosis was not colloid cyst, or surgical intervention was performed at an outside institution. On the basis of these criteria, 11 cases were excluded. Patients in the excluded group of cases had a mean age of 45.8 years and 2 of the patients in the group had died. One of the patients died of metastatic non-small cell lung cancer, and the other died due to complications of small bowel obstruction at the age of 91 . All study participants, including patients lost to follow-up, were queried in the National Death Index (NDI) to identify unreported or unexplained deaths within the cohort. There was 1 unexplained death, involving a 38-year-old woman with a $3-\mathrm{mm}$ colloid cyst that was identified incidentally 9 years previously.

Our retrospective cohort was composed of 163 cases of colloid cyst of the third ventricle diagnosed due to symptomatic presentation ( $\mathrm{n}=65,40 \%)$ or identified incidentally $(\mathrm{n}=98,60 \%)$ in patients undergoing evaluation for trauma, nonspecific headache, stroke, metabolic encephalopathy, or other symptoms for which neuroimaging was indicated (Table 1). Patients with colloid cysts remained in the incidental group if their symptoms were attributable to an alternative cause and no ventriculomegaly was identified on neuroimaging. Patients with established diagnoses 
TABLE 1. Presenting symptoms or indication for neuroimaging

\begin{tabular}{lcc}
\hline & \multicolumn{2}{c}{ No. $(\%)^{*}$} \\
\cline { 2 - 3 } Symptom or Indication & $58(89)$ & $24(25)$ \\
\hline Headache & $15(23)$ & $5(5)$ \\
\hline Nausea/vomiting & $14(22)$ & $2(2)$ \\
\hline Vision change & $13(20)$ & $6(6)$ \\
\hline Dizziness/ataxia & $9(14)$ & $11(11)$ \\
\hline Cognitive decline & $6(9)$ & $5(5)$ \\
\hline Syncope & $4(6)$ & $2(2)$ \\
\hline Seizure & $4(6)$ & $0(0)$ \\
\hline Bradycardia & $3(5)$ & $1(1)$ \\
\hline Sensory change & $3(5)$ & $0(0)$ \\
\hline Memory decline & $2(3)$ & $0(0)$ \\
\hline Urinary incontinence & $1(2)$ & $27(28)$ \\
\hline Trauma & $1(2)$ & $12(12)$ \\
\hline General medical & $1(2)$ & $3(3)$ \\
\hline Weakness & $0(0)$ & $8(8)$ \\
\hline General neurological & $0(0)$ & $8(8)$ \\
\hline Stroke & $0(0)$ & $6(6)$ \\
\hline Cancer staging & $0(0)$ & $2(2)$ \\
\hline Intoxication & $0(0)$ & $1(1)$ \\
\hline Other & $5 \%$ & \\
\hline & &
\end{tabular}

* The sum of the percentages is not $100 \%$, because multiple symptoms were present in most patients.

of congenital or normal pressure hydrocephalus without new or progressive symptoms and without new ventriculomegaly $(n=2)$ remained in the incidental group.

Univariate analysis of symptomatic versus incidental cases evaluated variables including age at presentation, presence of headache, cyst diameter, cyst volume $\left(4 / 3 \pi r_{1}\right.$ $\mathrm{r}_{2} \mathrm{r}_{3}$ ), signal intensity on several MRI sequences, and the presence of obstructive hydrocephalus (Table 2). Hydrocephalus was defined as ventriculomegaly on neuroimaging with the presence of 1 or more symptoms of elevated intracranial pressure, including headache, nausea with or without emesis, blurred vision or diplopia, dizziness or ataxic gait, cognitive decline, syncope, and bradycardia. The Evans ratio (frontal horn to maximum biparietal diameter) was calculated for all patients except those with a prior diagnosis of congenital hydrocephalus or with definite hydrocephalus ex vacuo, usually due to encephalomalacia from prior cerebral infarction. The symptomatic group was further subdivided and analyzed based on the presence of hydrocephalus $(n=30,46.2 \%)$ and acute hydrocephalus $(\mathrm{n}=8,12.3 \%)$. The latter was defined as hydrocephalus with symptom onset or acute progression from baseline within 24 hours of presentation. ${ }^{4}$ In addition, we defined third ventricle risk zones by dividing the structure into 3 regions based on the sagittal plane as seen on T1-weighted MRI (see Fig. 3): Zone I, from the lamina terminalis to a vertical line drawn from the mammillary body tangential to the massa intermedia; Zone II, from the posterior boundary of Zone I to the rostral apex of the midbrain tegmentum at the inlet of the cerebral aqueduct; Zone III, from the posterior boundary of Zone II to the posterior limit of the third ventricle at the posterior commissure. The term "risk zone" refers collectively to Zones I and III. Patients with incidentally discovered cysts who underwent serial neuroimaging ( $\mathrm{n}=57,58 \%$ of incidental cohort) were assessed for progression, defined as $>20 \%$ increase in cyst diameter with stable, new, or progressive symptoms of hydrocephalus, or regression, defined as similar magnitude decrease in diameter with stable or improved symptoms of hydrocephalus.

\section{Statistical Analysis}

All statistical analysis was performed using IBM SPSS version 21. Sample size required to achieve $80 \%$ power was estimated a priori using a noncentral t-distribution with estimated effect size $=0.4, \alpha=0.05$. For continuous variables, Levene's test for equality of variances was performed, followed by the Student t-test. Values of $\mathrm{p}<$ 0.05 were considered statistically significant. Continuous variables were dichotomized around critical values for relative risk (RR) calculations. Categorical variables were explored with the chi-square and Fischer exact tests. Statistically significant variables from univariate analyses were assessed for the ability to predict the outcome variables: symptomatic clinical status and obstructive hydrocephalus using binary logistic regression with and without bootstrapping. ${ }^{6}$ The Hosmer-Lemeshow test was used to assess goodness-of-fit, and cross-validation was performed using a 50/50 split technique. Binary classification models were assessed with receiver-operator characteristics.

\section{Results}

\section{Patient Presentation}

Symptomatic patients represented a clear minority of the overall cohort (65 patients [40\%] vs 98 [60\%] in the group with incidentally discovered colloid cysts). The most common presenting complaints included sequelae of hydrocephalus, such as headache, nausea/vomiting, blurred vision or diplopia, dizziness or ataxic gait, cognitive decline, and syncope (Table 1), consistent with prior case series. ${ }^{4,5,13-15}$ Indeed, hydrocephalus was common, occurring in $46.2 \%$ of symptomatic patients, 8 of whom presented acutely (12.3\%). Two patients transferred from outside hospitals with evidence of acute hydrocephalus and herniation on neuroimaging. One patient arrived in extremis; her condition did not improve following external ventricular drain placement, and she was pronounced brain dead. The second patient was transferred for neurological evaluation after multiple seizures followed by unresponsiveness and was also pronounced brain dead. There were no additional colloid cyst-related deaths in the present study.

\section{Incidental Group}

Incidentally discovered colloid cysts of the third ventricle were identified most frequently during routine neuroimaging for trauma (28\%); nonspecific headache (25\%); acute and chronic neurological conditions (20\%), such as stroke, peripheral neuropathy, and amyotrophic lateral sclerosis; and metabolic encephalopathy (12\%), often due to preexisting medical conditions. In the remaining cas- 
TABLE 2. Univariate comparison of incidental and symptomatic colloid cyst cohorts*

\begin{tabular}{|c|c|c|c|c|}
\hline Characteristic & Symptomatic & Incidental & p Value & $\operatorname{RR}(95 \% \mathrm{Cl})$ \\
\hline No. of patients & $65(39.9)$ & $98(60.1)$ & & \\
\hline Mean age in yrs & $37.9 \pm 15.5$ & $54.2 \pm 19.8$ & $<0.001$ & - \\
\hline Age $<65$ yrs & $60(96.9)$ & $59(70.4)$ & $<0.001$ & $7.40(1.91-28.60)$ \\
\hline Sex, F/M & $33 / 32$ & $38 / 60$ & 0.088 & $1.34(0.92-1.94)$ \\
\hline Mean cyst diameter in $\mathrm{mm}$ & $11.3 \pm 5.0$ & $7.0 \pm 3.0$ & $<0.001$ & - \\
\hline Cyst diameter $\geq 7 \mathrm{~mm}$ & $57(87.7)$ & $44(45.8)$ & $<0.001$ & $4.23(2.17-8.25)$ \\
\hline Mean cyst volume in $\mathrm{mm}^{3}$ & $1718.7 \pm 4194.4$ & $365.5 \pm 460.6$ & 0.021 & - \\
\hline Cyst volume $\geq 180 \mathrm{~mm}^{3}$ & $50(90.9)$ & $27(50.9)$ & $<0.001$ & $4.03(1.77-9.14)$ \\
\hline Mean Evans ratio & $0.31 \pm 0.07$ & $0.28 \pm 0.04$ & 0.003 & - \\
\hline Headache & $60(92.3)$ & $21(21.9)$ & $<0.001$ & $11.85(5.02-28.00)$ \\
\hline Obstructive hydrocephalus & $30(46.2)$ & 0 & $<0.001$ & $2.34(1.89-2.89)$ \\
\hline Acute hydrocephalus $\dagger$ & $8(12.3)$ & 0 & 0.009 & $2.04(1.74-2.40)$ \\
\hline Hyperattenuating CT & $47(92.2)$ & $83(95.4)$ & 0.333 & $0.72(0.35-1.50)$ \\
\hline \multicolumn{5}{|l|}{ Increased signal } \\
\hline $\mathrm{T} 1$ & $34(64.2)$ & $31(56.4)$ & 0.265 & $1.18(0.79-1.78)$ \\
\hline $\mathrm{T} 2$ & $25(50.0)$ & $19(34.5)$ & 0.080 & $1.39(0.93-2.06)$ \\
\hline FLAIR & $30(76.9)$ & $18(38.3)$ & $<0.001$ & $2.64(1.43-4.86)$ \\
\hline Contrast enhancement & $14(28.0)$ & $10(18.9)$ & 0.194 & $1.28(0.85-1.94)$ \\
\hline Decreased SWI signal & $3(8.8)$ & $6(20)$ & 0.285 & $0.59(0.23-1.53)$ \\
\hline Risk zone & $54(96.4)$ & $33(64.7)$ & $<0.001$ & $6.21(1.65-23.35)$ \\
\hline Surgical intervention & $59(90.8)$ & $3(3.1)$ & $<0.001$ & $16.02(7.36-34.88)$ \\
\hline Cyst-related death & $2(3.1)$ & 0 & - & - \\
\hline
\end{tabular}

FLAIR = fluid-attenuated inversion recovery; $S W I$ = susceptibility-weighted imaging.

* Values represent numbers of patients (\%) except where otherwise indicated. Means are presented \pm SD. Boldface type indicates statistical significance.

† Defined as hydrocephalus with symptom onset or acute progression from baseline within 24 hours of presentation (see Methods).

es, the indications for imaging included syncope, ataxia, dizziness or gait instability, seizures, and cancer staging (Table 1). Patients with incidental colloid cysts who were discharged from the emergency department were commonly lost to follow-up. Fifty-seven patients (58\%) with incidental colloid cysts underwent serial neuroimaging with a mean follow-up of 5.1 years (range $0.5-23$ years); 10 patients were followed for at least 10 years. Over the study period, 5 patients (8.8\%) followed with serial neuroimaging experienced progression and underwent resective surgery. No patient with an incidentally discovered, asymptomatic colloid cyst experienced acute obstructive hydrocephalus or sudden neurological deterioration in the absence of antecedent trauma. Interestingly, 2 patients (3.5\%) demonstrated cyst regression, both with greater than $30 \%$ decrease in diameter of their lesions. In 1 case, the spheroid volume of the lesion decreased nearly 5 -fold, from $725 \mathrm{~mm}^{3}$ to $150 \mathrm{~mm}^{3}$. Three patients with incidental colloid cysts underwent elective resection. One patient had a 14-mm $\left(806-\mathrm{mm}^{3}\right)$ cyst discovered after evaluation for chronic alcoholism. A second patient underwent a CT scan of the head for chronic sinusitis and was found to have a $13-\mathrm{mm}\left(1525-\mathrm{mm}^{3}\right)$ colloid cyst and ventriculomegaly. The third patient was assaulted and underwent imaging that demonstrated an $8-\mathrm{mm}\left(268-\mathrm{mm}^{3}\right)$ colloid cyst with ventriculomegaly. Although the patient complained of no symptoms, he demonstrated marked sinus bradycardia while supine. Cardiac evaluation revealed no underlying cause, and the patient subsequently underwent resection. Notably, the patient's bradycardia resolved fully following surgery.

\section{Symptomatic Group}

Nearly all patients with symptomatic colloid cysts underwent resection $(n=59,91 \%)$. Significant differences were found with respect to several observed demographic, morphological, and neuroimaging features when patients with symptomatic colloid cysts were compared to patients with incidental cysts (Table 2). These features included age at presentation (37.9 years vs 54.2 years), proportion of patients with age $<65$ years (96.9\% vs 70.4\%, RR 7.4), headache on presentation ( $92.3 \%$ vs $21.9 \%$, RR 11.9), cyst diameter (11.3 mm vs $7.0 \mathrm{~mm}$ ) (Fig. 1 left), proportion of patients with cyst diameter $\geq 7 \mathrm{~mm}(87.7 \%$ vs $45.8 \%$, RR $4.2)$, cyst volume $\left(1718.7 \mathrm{~mm}^{3}\right.$ vs $\left.365.5 \mathrm{~mm}^{3}\right)$, proportion of patients with cyst volume $\geq 180 \mathrm{~mm}^{3}$ (90.9\% vs 50.9 , RR 4.0), cyst hyperintensity on FLAIR MRI sequences (76.9\% vs 38.3\%, RR 2.6) (Fig. 2), and the proportion of patients with hydrocephalus ( $46.2 \%$ vs $0 \%)$. These results are consistent with the previous study by Pollock et al., with the exception that cyst hyperintensity on T2-weighed MRI did not achieve statistical significance in the present study $(50.0 \%$ vs $34.5 \%, \mathrm{p}=0.080) .{ }^{15}$ Interestingly, the proportion of symptomatic patients with cyst hyperintensity on FLAIR MRI sequences was highly significant when 


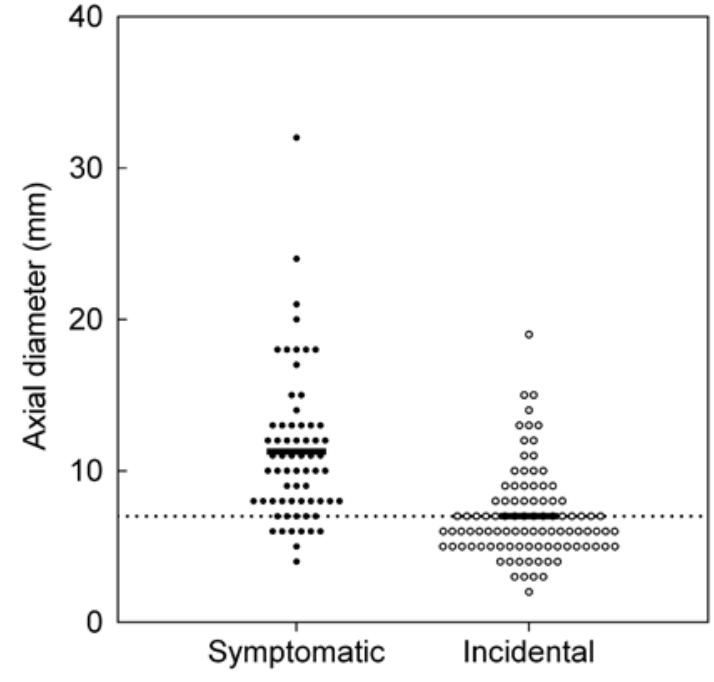

Clinical status

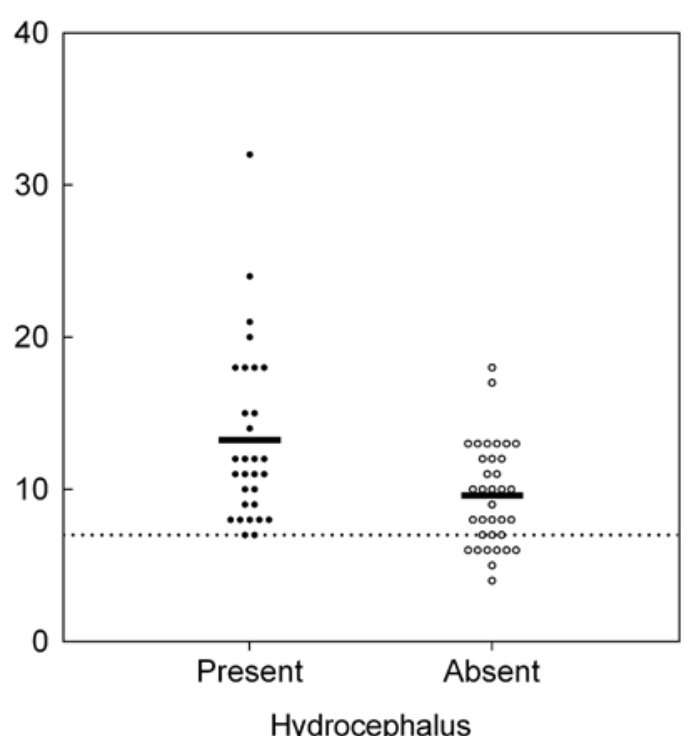

Hydrocephalus

FIG. 1. Scatter plots of colloid cyst axial diameters. Left: Patients with symptomatic colloid cysts demonstrated a wide range of axial diameter, while the majority of those with incidental cysts (86.7\%) had diameter $\geq 7 \mathrm{~mm}$ (dashed line). The mean axial diameter differed significantly between the 2 groups $(11.3 \pm 5.0 \mathrm{~mm}$ vs $7.0 \pm 3.0 \mathrm{~mm}$, respectively, $p<0.0001$, solid bars). Right: Symptomatic patients with hydrocephalus had colloid cysts with axial diameter $\geq 7 \mathrm{~mm}$ (dashed line). The mean axial diameter of these lesions was significantly greater than that of the lesions in symptomatic patients without hydrocephalus $(13.2 \pm 5.8 \mathrm{~mm}$ and $9.6 \pm 3.4 \mathrm{~mm}$, respectively, $\mathrm{p}<0.004$, solid bars).

compared with patients with incidental cysts. This may be due to the increased sensitivity of the FLAIR sequence for tissue edema, compared with conventional T2-weighted sequences. The mean Evans ratio for symptomatic patients slightly exceeded the threshold suggestive of hydrocephalus and was significantly different when compared with patients with incidental cysts $(0.31$ vs $0.28, \mathrm{p}=0.003)$.

\section{Risk Factors for Hydrocephalus}

Nearly one-half of symptomatic patients with colloid cyst of the third ventricle presented with hydrocephalus ( $\mathrm{n}=30,46.2 \%)$, and all except 2 patients that presented with herniation and brain death underwent neurosurgical intervention. Several of the statistically significant factors identified when symptomatic patients were compared with those with incidental lesions remained significant upon univariate comparison of the subset of symptomatic patients with and without hydrocephalus. These included mean cyst diameter $(13.2 \mathrm{~mm}$ vs $9.6 \mathrm{~mm})$, proportion of patients with cyst diameter $\geq 7 \mathrm{~mm}(100 \%$ vs $77.1 \%$, RR 4.7) (Fig. 1 right), proportion of patients with cyst volume $\geq 180 \mathrm{~mm}^{3}$ (100\% vs $82.8 \%$, RR 3.1), Evans ratio (0.36 vs 0.27 ), and cyst hyperintensity on T2-weighted (70.0\% vs $36.7 \%$, RR 2.3) and FLAIR MRI (94.4\% vs $61.9 \%$, RR 5.1). In all but 1 of the patients with hydrocephalus, the colloid cyst was located in Zone I, the classic location within the foramen of Monro at the anterior margin of the third ventricle (Fig. 3A and B). Importantly, no patient with a Zone II lesion (Fig. 3C) presented with hydrocephalus or progressive symptoms in the present study. A single patient with rapidly progressive cognitive decline and acute obstructive hydrocephalus was found to have a Zone III lesion obstructing the cerebral aqueduct (Fig. 3D). The proportion of patients with signal hypointensity on susceptibility-weighted imaging (SWI) $(22.0 \%$ vs
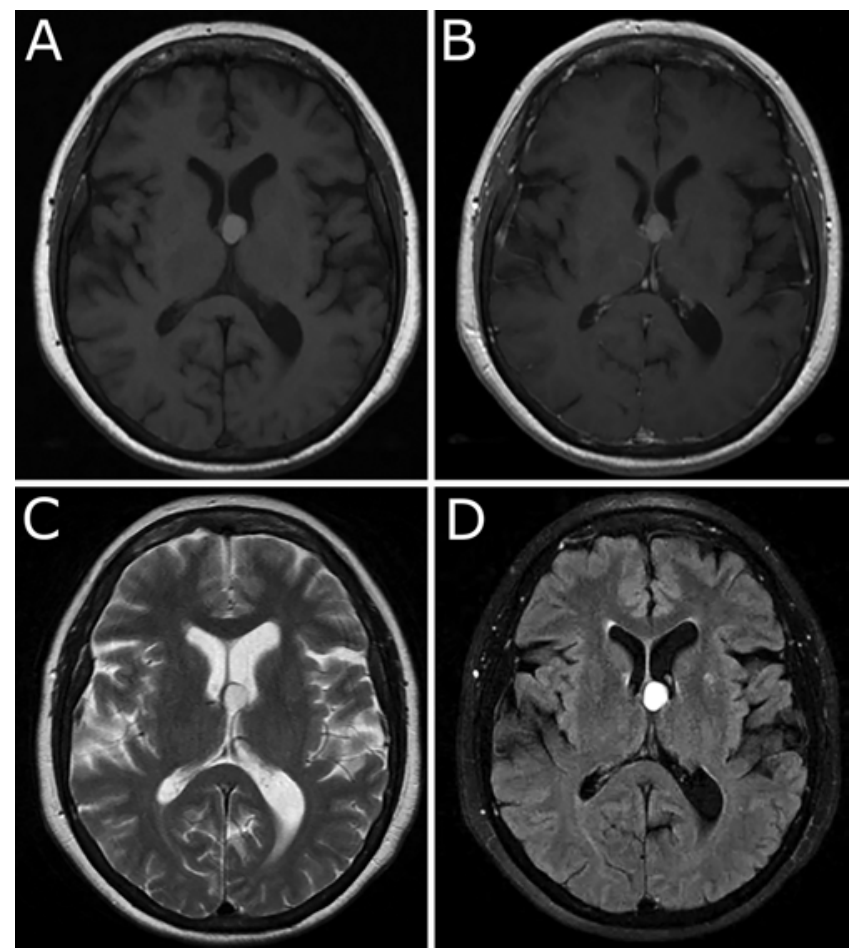

FIG. 2. Neuroimaging of a symptomatic colloid cyst. A and B: Axial noncontrast (A) and postcontrast (B) T1-weighted MR images demonstrating a well-circumscribed 8-mm lesion in the foramen of Monro. The lesion is hyperintense and without enhancement. C: Axial T2-weighted MR image. T2-weighted sequences also demonstrated cyst hyperintensity and mild asymmetrical ventriculomegaly involving the left lateral ventricle. D: Axial FLAIR MR image demonstrating marked cyst hyperintensity without periventricular edema. 


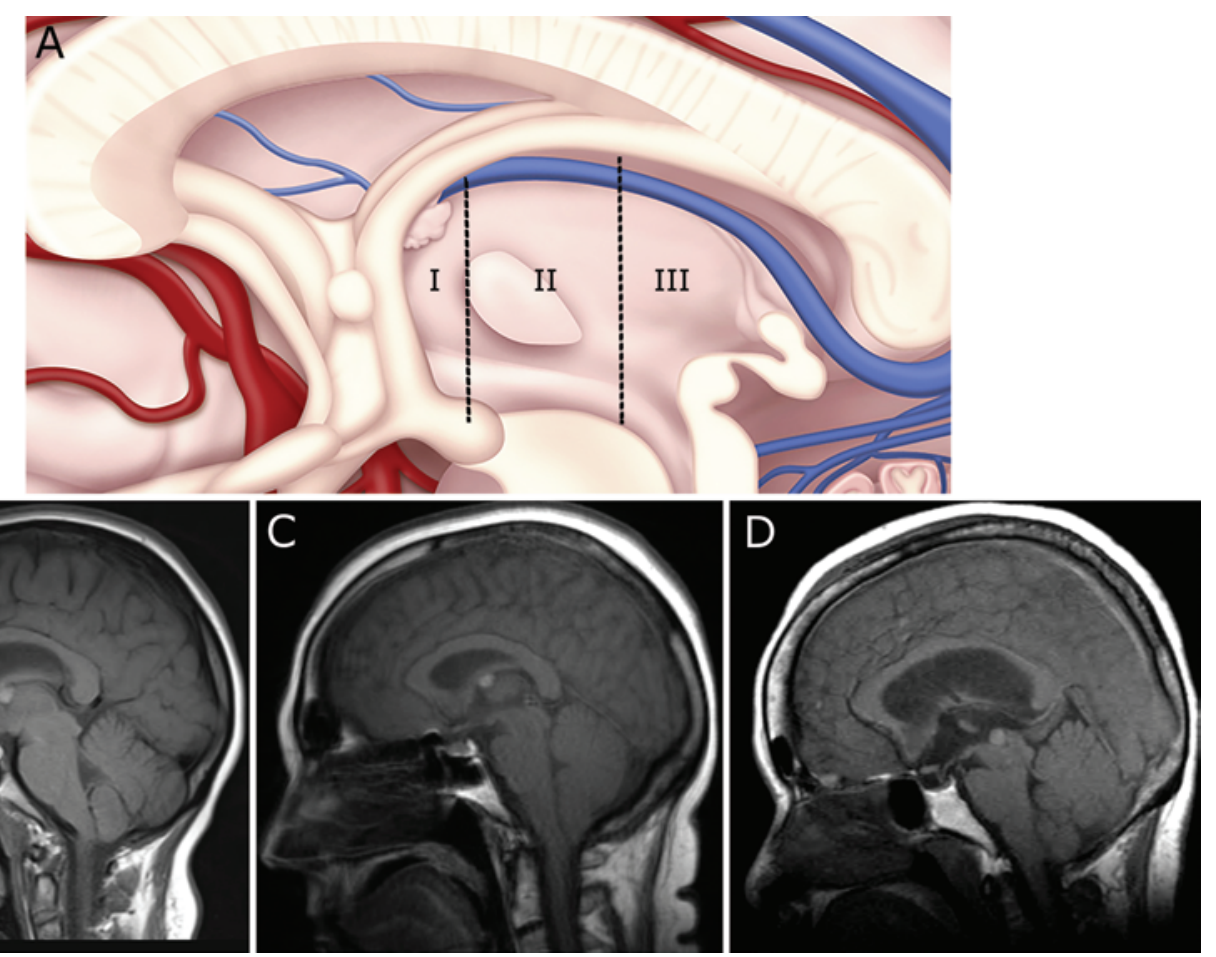

FIG. 3. Anatomical risk zones for colloid cysts of the third ventricle. A: The third ventricle was divided into 3 zones along the sagittal axis: Zone I, from the lamina terminalis to a vertical line drawn from the mammillary body and tangential to the massa intermedia; Zone II, from the posterior boundary of Zone I to the rostral apex of the midbrain tegmentum at the inlet of the cerebral aqueduct; Zone III, from the posterior boundary of Zone II to the posterior limit of the third ventricle. The term "risk zone" refers collectively to Zones I and III. Copyright Thomas L. Beaumont. Published with permission. B-D: Sagittal noncontrast T1-weighted MR images demonstrating Zone I (B), II (C), and III (D) lesions.

$12.7 \%, \mathrm{p}=0.602$ ) was not significantly associated with the development of obstructive hydrocephalus.

Both colloid cyst diameter and volume were strongly associated with obstructive hydrocephalus. There was a relatively wide distribution of ellipsoid volumes within a given axial diameter group (Fig. 4A and B). Interestingly, for several axial diameter groups $\geq 7 \mathrm{~mm}$, the largest volume cysts in the group were not associated with CSF obstruction, while smaller volume lesions were seen in patients who presented with hydrocephalus (Fig. 4B). In at least 2 diameter groups, symptomatic cysts causing obstructive hydrocephalus had volumes 1.5- to 3.7-fold less than lesions that did not result in obstructive hydrocephalus. Most striking was the 14 -fold difference between the largest volume colloid cyst without associated obstructive hydrocephalus $\left(3562.5 \mathrm{~mm}^{3}\right)$ and the smallest volume lesion that did result in obstructive hydrocephalus (249.2 $\mathrm{mm}^{3}$ ). Further analysis of these particular cases demonstrated smaller volume cysts that caused hydrocephalus commonly projected caudally through the foramen of Monro into the third ventricle (Fig. 4C). In contrast, cysts with comparatively larger volumes that did not cause hydrocephalus often projected rostrally from the foramen of Monro into the lateral ventricle and had large coronal diameters relative to their axial diameter (i.e., prolate spheroid) (Fig. 4D).

\section{Acute Hydrocephalus and Death}

Eight patients presented with acute hydrocephalus, de- fined as acute progression of baseline symptoms or onset of new, rapidly progressive symptoms of hydrocephalus within 24 hours of presentation. These patients represented $12.3 \%$ of the symptomatic group and $4.9 \%$ of all patients in the study. No significant difference in age, cyst diameter, or cyst volume was observed between symptomatic patients with and without acute hydrocephalus. Importantly, $50 \%$ of the patients presenting with acute hydrocephalus experienced the onset of new, progressive symptoms over 3-4 days prior to acute neurological deterioration. One such patient had a 1-month history of headaches with a progressive increase in severity over 4 days preceding presentation and subsequently experienced cognitive decline and somnolence while undergoing MRI. In an extreme example, a patient with a 4-day history of progressive headache was transferred from an outside hospital in extremis, with evidence of acute hydrocephalus and herniation on neuroimaging. The patient's condition did not improve following external ventricular drain placement and ultimately met criteria for brain death. Similarly, a second patient with a known colloid cyst was transferred from an outside hospital after multiple seizures followed by apnea and bradycardia. The patient demonstrated ventriculomegaly and herniation on imaging, and examination findings were consistent with brain death. Thus, these data suggest that the risk of death in symptomatic patients with a colloid cyst of the third ventricle is $3.1 \%$.

Two additional cases of acute hydrocephalus are noteworthy. The first involved a teenager who experienced a 

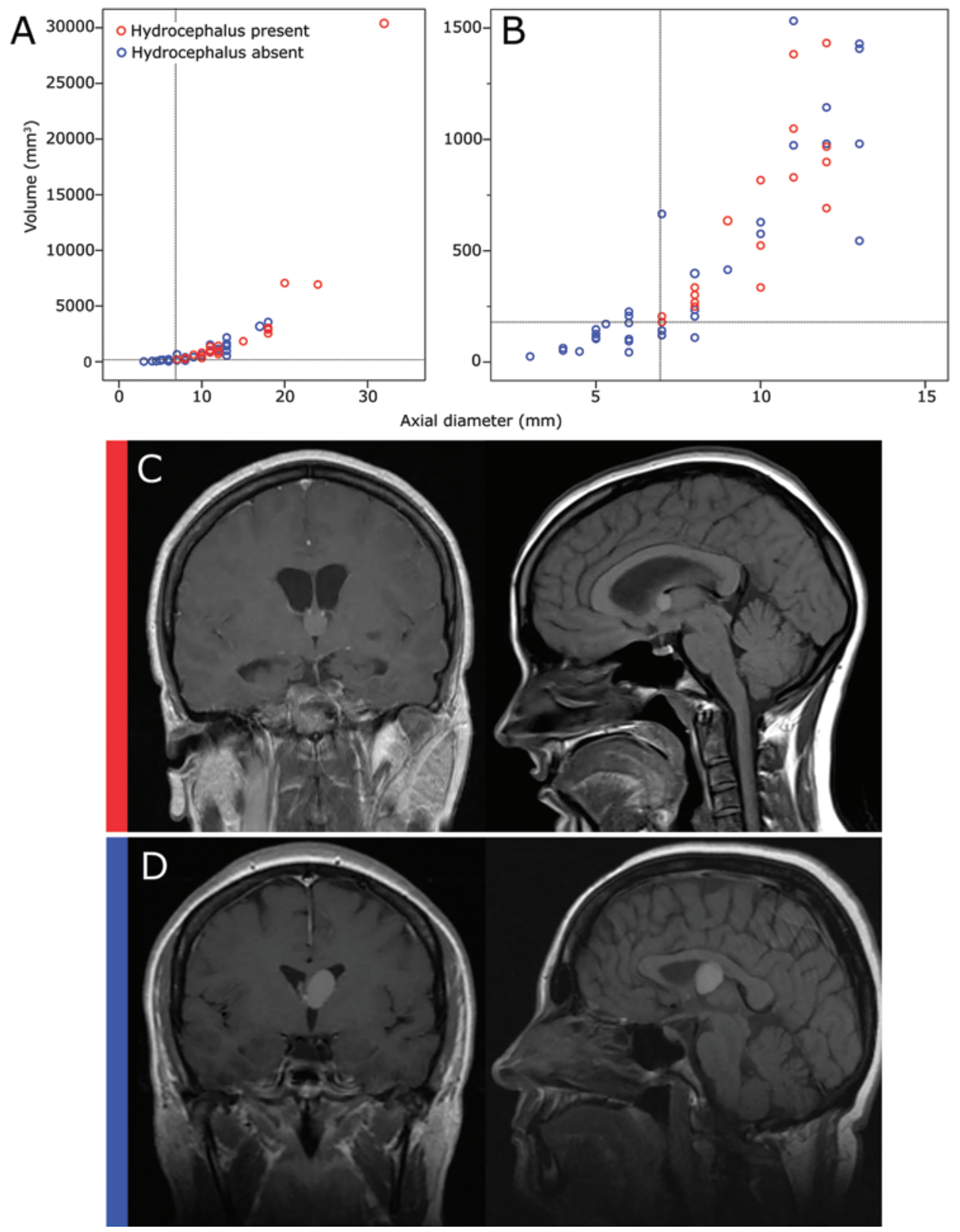

FIG. 4. Relationship of colloid cyst diameter to volume. A: Plot of diameter versus volume for colloid cysts with and without hydrocephalus (red and blue circles, respectively). B: Expanded plot for cysts $\leq 15 \mathrm{~mm}$ demonstrated a wide range of volumes within several diameter groups. Larger volume cysts were often not associated with obstructive hydrocephalus. In at least 2 groups, symptomatic cysts causing obstructive hydrocephalus had cyst volumes up to 3.7-fold less than lesions of the same axial diameter that did not result in obstructive hydrocephalus. C: Cysts with smaller spheroid volumes that projected inferiorly through the foramen of Monro into the third ventricle commonly resulted in obstructive hydrocephalus. D: Cysts with large coronal diameters relative to their axial diameter often projected superiorly into the lateral ventricle and were not associated with obstructive hydrocephalus.

concussion with 2-3 minutes loss of consciousness while playing organized football. Neuroimaging demonstrated an $18-\mathrm{mm}$ colloid cyst without ventriculomegaly. Over the subsequent 12 hours the patient experienced severe, rapidly progressive headache and developed new ventriculomegaly on repeat neuroimaging. In the second case, a patient with a known asymptomatic colloid cyst of the third ventricle complained of slowly progressive gait instability after a fall from standing 1 week prior, with subsequent progressive cognitive decline over 24 hours. Neuroimaging demonstrated a hyperintense lesion obstructing the cerebral aqueduct with a well-defined, hypointense region at the foramen of Monro (Fig. 3D). These findings were best explained by suggesting the colloid cyst (initially diagnosed at an outside hospital) originating near the foramen of Monro had detached and translocated to the dependent portion of the posterior third ventricle. ${ }^{16,19}$ This was the only Zone III lesion observed in the study, as all the other 
cases of acute hydrocephalus involved lesions in Zone I. Taken together, these 2 cases suggest traumatic translocation as a rare, but possible mechanism of acute obstructive hydrocephalus in patients with colloid cysts of the third ventricle.

\section{Colloid Cyst Severity Score}

The most significant risk factors for symptomatic colloid cysts identified on univariate testing were age at presentation, presence of headache, cyst diameter $\geq 7 \mathrm{~mm}$, cyst hyperintensity on FLAIR MRI, and cyst location in an anatomical risk zone. These variables were subsequently combined and assessed for their predictive capacity using logistic regression. We elected to use axial cyst diameter instead of cyst volume, as these 2 variables had similarly strong statistical association with symptomatic lesions and the former does not require orthogonal imaging and volume computation. The model demonstrated significant predictive capacity, achieving sensitivity and specificity of $92 \%$ and $93 \%$, respectively, for symptomatic clinical status, and $88 \%$ and $82 \%$ for the subset of symptomatic patients presenting with obstructive hydrocephalus. These risk factors were therefore combined into a simple 5-point risk score herein referred to as the Colloid Cyst Risk Score (CCRS) (Table 3). Further analysis of the CCRS with receiver-operator characteristics again demonstrated significant predictive capacity with area under the curve (AUC) of 0.917 for symptomatic patients and 0.845 for patients presenting with hydrocephalus (Fig. 5). The CCRS provided statistically significant stratification of symptomatic patients across the 5 CCRS levels, ranging from $5.9 \%$ in CCRS 2 to $96.0 \%$ in CCRS $5\left(\chi^{2}<0.0001\right)$ (Table 4). A similar statistically significant relationship was seen for patients presenting with hydrocephalus; $20.0 \%$ for CCRS 4 ranging up to $60.0 \%$ for CCRS 5 . Notably, all patients presenting with hydrocephalus had CCRS $\geq 4$, which demonstrated strong statistical association with hydrocephalus ( $p<0.0001$, RR 19.4). The only patients in the incidental group who experienced progression over the study period were those with CCRS $\geq 3$ based on initial imaging. Taken together, these data suggest that patients with CCRS $\geq 4$ represent the high-risk subgroup, while CCRS $\leq 2$ represents the low-risk subgroup. CCRS 3 can be considered intermediate risk, as 3 of the 5 patients in the incidental group who demonstrated progression on surveillance neuroimaging had CCRS of 3 on initial presentation, but progressed to CCRS 5 within 5 years. Nevertheless, as progression was observed in relatively few patients $(\mathrm{n}=$ $5)$, external validation will be necessary to corroborate the predictive value of the CCRS.

\section{Discussion}

Specific surgical indications for colloid cysts of the third ventricle have remained undefined due to the rarity of the lesion in the general population and the associated inability to conduct randomized controlled trials. As a result, decision making in the management of colloid cysts is most commonly driven by individual clinicians' judgment, based on general neurosurgical experience and published case series, most with limited patient numbers.
TABLE 3. Colloid Cyst Risk Score

\begin{tabular}{cc}
\hline Criterion & Points \\
\hline Age $<65$ yrs & 1 \\
\hline Yes & 0 \\
\hline No & \\
\hline Headache & 1 \\
\hline Yes & 0 \\
\hline No & \\
\hline Axial diameter $\geq 7 \mathrm{~mm}$ & 1 \\
\hline Yes & 0 \\
\hline No & 1 \\
\hline FLAIR hyperintensity & 0 \\
\hline Yes & 1 \\
\hline No & 0 \\
\hline Risk zone &
\end{tabular}

Given the commonly cited risk of sudden deterioration and death (ranging from 5\% to 12\%) and that colloid cysts are often identified in otherwise healthy young adults, clinicians have been forced to weigh the cumulative risk of sudden death against the risks associated with resection. Although colloid cysts are histologically benign lesions, their location deep within the brain, in association with the fornix, presents significant risk of major complications during surgical removal, approaching $15 \%$ on average. ${ }^{18}$ Interestingly, in the present study, no patient with an incidentally discovered, asymptomatic colloid cyst of the third ventricle experienced acute obstructive hydrocephalus, sudden neurological deterioration, or death. Five patients $(8.8 \%)$ followed with serial neuroimaging demonstrated radiological and/or symptomatic progression; 2 of which underwent urgent surgical excision due to new ventriculomegaly, whereas the remaining 3 underwent surgery on an elective basis. Notably, each of these patients presented with a progressive increase in baseline headaches, with the 2 patients who presented acutely experiencing new symptoms, including blurred vision, memory decline, and gait instability. These data are consistent with more recent studies demonstrating that nearly all patients with acute obstructive hydrocephalus have progressive symptoms for more than 24 hours preceding precipitous clinical deterioration. ${ }^{2,10}$ The rare exceptions involve instances of acute hemorrhage within the colloid cyst, referred to as "cyst apoplexy." 7,10 This may cause rapid expansion of the lesion with acute obstructive hydrocephalus and precipitous neurological decline. In the 1 case of cyst apoplexy identified in the present study, the patient described having new holocephalic headaches for 2 weeks followed by progressive cognitive decline, nausea, and vomiting over 3 days prior to presentation. Neuroimaging demonstrated a hyperintense lesion on FLAIR imaging with blooming hypointensity on susceptibility-weighted imaging. Gross blood products were seen within the cyst at surgery and on pathological examination. Similarly, in 1 of the 2 cases of colloid cystrelated death due to obstructive hydrocephalus and hernia- 

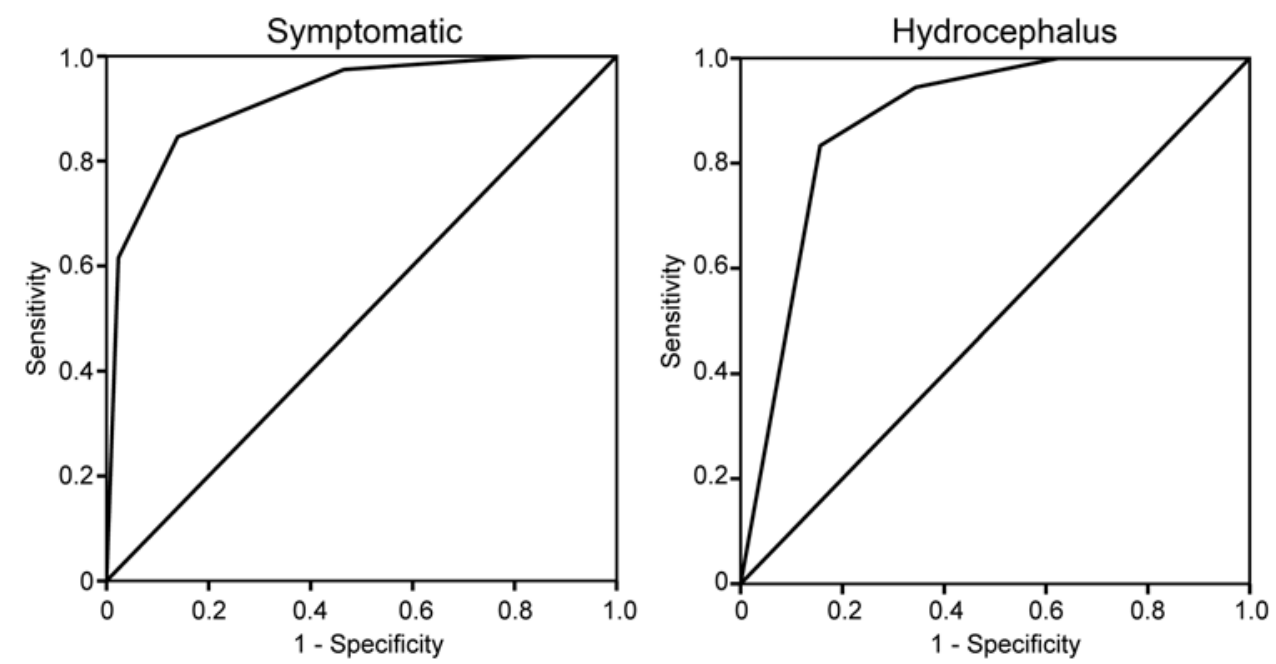

FIG. 5. Receiver-operator curves for the Colloid Cyst Risk Score (CCRS) demonstrating significant predictive capacity. The AUC was 0.917 for symptomatic colloid cysts (left) and 0.845 for cysts presenting with hydrocephalus (right). The CCRS identifies symptomatic lesions with sensitivity and specificity of $92 \%$ and $93 \%$, respectively, and $88 \%$ and $82 \%$ for the subset of patients presenting with hydrocephalus.

tion, the patient experienced 4 days of severe, progressive headache followed by nausea, vomiting, and obtundation the evening prior to presentation. The subacute onset of symptoms followed by acute decline in these cases raises the question of whether intralesional hemorrhage leads directly to rapidly progressive symptoms or alternatively, whether hemorrhage occurs at the time of symptom onset followed by osmotic flow of fluid into the lesion resulting in cyst expansion and progressive obstruction. Nevertheless, these data indicate that while $12.3 \%$ of symptomatic patients with colloid cysts may experience acute obstructive hydrocephalus, death is less frequent, occurring in $3.1 \%$ of symptomatic patients, $1.2 \%$ overall.

As a primary aim of the study, we confirmed several previously identified risk factors associated with symptomatic colloid cysts and with the development of cystrelated obstructive hydrocephalus. These factors include younger age $(<65$ years), the presence of headache, and cyst diameter. ${ }^{3,5,13,14}$ We identified 3 additional factors not previously reported, including cyst volume, hyperintensity on FLAIR imaging, and cyst location in a third ventricular risk zone. In their study of the natural history of colloid cysts, Pollock et al..$^{15}$ reported that colloid cysts with increased signal on $\mathrm{T} 2$-weighted sequences had a 5-fold increased likelihood of being symptomatic when compared with asymptomatic lesions. Further, increased sig-

TABLE 4. Patient stratification based on Colloid Cyst Risk Score

\begin{tabular}{ccc}
\hline & \multicolumn{3}{c}{ No. (\%) } \\
\cline { 2 - 3 } CCRS & Symptomatic & Hydrocephalus \\
\hline 0 & 0 & 0 \\
\hline 1 & 0 & 0 \\
\hline 2 & $1(6)$ & 0 \\
\hline 3 & $4(22)$ & 0 \\
\hline 4 & $10(67)$ & $3(20)$ \\
\hline 5 & $24(96)$ & $15(60)$ \\
\hline
\end{tabular}

nal on T2-weighted sequences was one of 5 factors that defined the Class III patient group, which had the greatest likelihood of presenting with obstructive hydrocephalus. Although hyperintensity on T2-weighted imaging did not demonstrate a statistically significant association with symptomatic colloid cysts in the present study, it was significantly associated with the subgroup presenting with hydrocephalus ( $p=0.021, \mathrm{RR} 2.3)$. On the other hand, there was a strong association of cyst hyperintensity on FLAIR imaging in patients with symptomatic lesions $(p<0.000$, RR 2.6), and in those presenting with obstructive hydrocephalus ( $p=0.019$, RR 5.1). Notably, with the exception of a single case, all patients with obstructive hydrocephalus demonstrated hyperintensity on FLAIR sequences. Thus, FLAIR hyperintensity represents a powerful new risk factor for symptomatic colloid cysts and for obstructive hydrocephalus in symptomatic patients. In our series, the signal intensity on FLAIR imaging was a stronger predictor of obstructive hydrocephalus than signal intensity on conventional T2-weighted sequences. This observation may be due to "protein doping" of the cyst fluid, such as occurs with hemorrhage or osmotic concentration, which is more readily appreciated on FLAIR sequences than on conventional T2-weighted sequences.

Although our results showed a strong association of the diameter of colloid cysts with the likelihood of the lesion causing symptoms $(11.3 \pm 5.0 \mathrm{~mm}$ vs $7.0 \pm 3.0 \mathrm{~mm}$, p < 0.000 , symptomatic vs incidental, respectively) and presentation with hydrocephalus $(13.2 \pm 5.8 \mathrm{~mm}$ vs $9.6 \pm 3.4$ $\mathrm{mm}, \mathrm{p}=0.004$, present vs absent, respectively), the relationship is likely multifactorial, involving several interacting factors..$^{15}$ The data presented here indicate that the risk of obstructive hydrocephalus begins at a cyst diameter of $7 \mathrm{~mm}$ as measured on axial T1-weighted MRI. No patient with a lesion diameter $<7 \mathrm{~mm}$ presented with symptoms of obstructive hydrocephalus (Fig. 1 right) or experienced progression during serial neuroimaging. The threshold effect seen here is consistent with prior reports suggest- 
ing that 7-mm asymptomatic colloid cysts be managed nonoperatively with surveillance imaging. ${ }^{11}$ On the other hand, our data are in direct contrast to the recent study by Margetis et al. that reported neither patient age nor cyst diameter was correlated with the absence or presence of symptoms in patients with colloid cysts. ${ }^{12}$ Although the ages of our symptomatic cohorts were similar $(37.9 \pm 15.5$ vs $43.3 \pm 15.7$ years), the incidental cohort was significantly older in the present study $(54.2 \pm 19.8$ vs $39.7 \pm 14.5$ years), which may account for the significant difference in age that we observed. This discrepancy could be explained by differences in the demographics of the respective regions (St. Louis, Missouri vs New York City), differences in referral patterns, and the fact that our institution is a regional trauma center that serves both an urban and rural catchment area.

We defined anatomical risk zones within the third ventricle to better understand the role of cyst location and diameter in the development of hydrocephalus. Remarkably, all but one of the symptomatic patients with hydrocephalus had a colloid cyst in Zone I, the classic location at the anterior extreme of the third ventricle. In contrast, none of the 21 patients with Zone II lesions (posterior to mass intermedia and anterior to the inlet of the cerebral aqueduct) presented with obstructive hydrocephalus. The single exception to the above findings was the case of acute hydrocephalus likely due to traumatic translocation of the cyst from the site of origin near the foramen of Monro to the inlet of the cerebral aqueduct. ${ }^{16,19}$ Thus, the posterior extreme of the third ventricle (Zone III) was also included as a risk zone, although it represents an exceedingly uncommon location for colloid cysts.

Several factors, including age $<65$ years, presence of headache on presentation, cyst diameter $\geq 7 \mathrm{~mm}$, hyperintensity on FLAIR imaging, and the location of the colloid cyst within a third ventricle risk zone, were significantly associated with symptomatic colloid cysts on univariate analysis and are likely clinically relevant given relative risk ranging from 2.6 to 11.9 (Table 2). Importantly, when these factors were combined in multivariate analysis, they remained statistically significant and demonstrated appreciable capacity for predicting symptomatic lesions with sensitivity and specificity of $92 \%$ and $93 \%$, respectively, for symptomatic clinical status, and $88 \%$ and $82 \%$ for the subset of patients presenting with hydrocephalus. These factors were therefore combined into a simple tool, the Colloid Cyst Risk Score (CCRS) that can be used to objectively identify symptomatic lesions and stratify the risk of obstructive hydrocephalus. As the present study is retrospective and few incidental lesions progressed during follow-up, the CCRS will require prospective external validation of its predictive capacity before being used as a framework on which management standards can be established. Nevertheless, patients with CCRS $\geq 4$ are considered to be at high risk for lesion progression and obstructive hydrocephalus.

In the incidental cohort, trauma was the most common indication for neuroimaging, accounting for one-fourth of cases. Although the majority of patients with incidentally discovered cysts had small asymptomatic lesions that did not progress on surveillance neuroimaging, 2 patients
( $2.0 \%$ of incidental cohort) experienced the acute onset of symptoms following trauma. This may be due to a traumainduced ball-valve effect, as a result of translocation of the lesion or compression of an already compromised foramen of Monro due to surrounding cerebral edema. An alternative mechanism could involve traumatic shearing forces severing the cyst attachment at the fornix, allowing translocation to the inlet of the cerebral aqueduct and thereby causing acute obstructive hydrocephalus. This mechanism may account for the single case of acute obstructive hydrocephalus due to a Zone III cyst described above, and it raises the possibility that trauma may convert an otherwise unknown incidental colloid cyst to a symptomatic lesion with acute obstructive hydrocephalus.

\section{Conclusions}

Colloid cysts of the third ventricle may undergo expansion resulting in symptom progression $(8.8 \%)$ or, less commonly, may undergo regression (3.5\%). In the present study, no patient with an incidentally discovered, asymptomatic colloid cyst experienced acute obstructive hydrocephalus or sudden neurological deterioration in the absence of antecedent trauma. Hydrocephalus was common, occurring in nearly one-half $(46.2 \%)$ of patients with symptomatic colloid cysts. The risk of death in symptomatic patients was $3.1 \%$. Several risk factors for symptomatic lesions were identified, including age $<65$ years, presence of headache on presentation, cyst diameter $\geq 7 \mathrm{~mm}$ on axial imaging, hyperintensity on FLAIR MRI, and location of the colloid cyst within a third ventricle risk zone. The Colloid Cyst Risk Score (CCRS) can be used to identify symptomatic lesions and stratify the risk of obstructive hydrocephalus during clinical decision making. External validation of the CCRS will be necessary before objective surgical indications can be established. Nevertheless, surgical intervention should be considered for all patients with $\mathrm{CCRS} \geq 4$, as they represent the high-risk subgroup.

\section{Acknowledgments}

We thank Matthew Holt (www.bodyrender.com) for the original artwork in Fig. 3A.

\section{References}

1. Boogaarts HD, Decq P, Grotenhuis JA, Le Guérinel C, Nseir $\mathrm{R}$, Jarraya B, et al: Long-term results of the neuroendoscopic management of colloid cysts of the third ventricle: a series of 90 cases. Neurosurgery 68:179-187, 2011

2. Büttner A, Winkler PA, Eisenmenger W, Weis S: Colloid cysts of the third ventricle with fatal outcome: a report of two cases and review of the literature. Int J Legal Med 110:260 266, 1997

3. Camacho A, Abernathey CD, Kelly PJ, Laws ER Jr: Colloid cysts: experience with the management of 84 cases since the introduction of computed tomography. Neurosurgery 24:693-700, 1989

4. de Witt Hamer PC, Verstegen MJ, De Haan RJ, Vandertop WP, Thomeer RT, Mooij JJ, et al: High risk of acute deterioration in patients harboring symptomatic colloid cysts of the third ventricle. J Neurosurg 96:1041-1045, 2002

5. Desai KI, Nadkarni TD, Muzumdar DP, Goel AH: Surgical management of colloid cyst of the third ventricle-a study of 105 cases. Surg Neurol 57:295-304, 2002 
6. Efron B, Tibshirani R: An Introduction to the Bootstrap. New York: Chapman \& Hall, 1993

7. Godano U, Ferrai R, Meleddu V, Bellinzona M: Hemorrhagic colloid cyst with sudden coma. Minim Invasive Neurosurg 53:273-274, 2010

8. Hernesniemi J, Leivo S: Management outcome in third ventricular colloid cysts in a defined population: a series of 40 patients treated mainly by transcallosal microsurgery. Surg Neurol 45:2-14, 1996

9. Horn EM, Feiz-Erfan I, Bristol RE, Lekovic GP, Goslar PW, Smith KA, et al: Treatment options for third ventricular colloid cysts: comparison of open microsurgical versus endoscopic resection. Neurosurgery 60:613-620, 2007

10. Jeffree RL, Besser M: Colloid cyst of the third ventricle: a clinical review of 39 cases. J Clin Neurosci 8:328-331, 2001

11. Kondziolka D, Lunsford LD: Stereotactic management of colloid cysts: factors predicting success. J Neurosurg 75:4551, 1991

12. Margetis K, Christos PJ, Souweidane M: Endoscopic resection of incidental colloid cysts. J Neurosurg 120:1259-1267, 2014

13. Mathiesen T, Grane P, Lindgren L, Lindquist C: Third ventricle colloid cysts: a consecutive 12-year series. J Neurosurg 86:5-12, 1997

14. Pollock BE, Huston J III: Natural history of asymptomatic colloid cysts of the third ventricle. J Neurosurg 91:364-369, 1999

15. Pollock BE, Schreiner SA, Huston J III: A theory on the natural history of colloid cysts of the third ventricle. Neurosurgery 46:1077-1083, 2000

16. Rosenstengel C, Baldauf J, Müller JU, Schroeder HW: Sudden intraaqueductal dislocation of a third ventricle ependymoma causing acute decompensation of hydrocephalus. $\mathbf{J}$ Neurosurg Pediatr 8:154-157, 2011
17. Ryder JW, Kleinschmidt-DeMasters BK, Keller TS: Sudden deterioration and death in patients with benign tumors of the third ventricle area. J Neurosurg 64:216-223, 1986

18. Sampath R, Vannemreddy P, Nanda A: Microsurgical excision of colloid cyst with favorable cognitive outcomes and short operative time and hospital stay: operative techniques and analyses of outcomes with review of previous studies. Neurosurgery 66:368-375, 2010

19. Zabihyan S, Etemadrezaie H, Baharvahdat H, Baradaran A, Ganjeefar B, Bohl MA, et al: Remote transplantation of a third ventricle colloid cyst: case report. J Neurosurg 122:1406-1410, 2015

\section{Disclosures}

The authors report no conflict of interest concerning the materials or methods used in this study or the findings specified in this paper.

\section{Author Contributions}

Conception and design: Beaumont, Wippold, Dacey. Acquisition of data: Beaumont. Analysis and interpretation of data: all authors. Drafting the article: Beaumont, Dacey. Critically revising the article: all authors. Reviewed submitted version of manuscript: all authors. Approved the final version of the manuscript on behalf of all authors: Beaumont. Statistical analysis: Beaumont. Administrative/technical/material support: Beaumont. Study supervision: Wippold, Dacey.

\section{Correspondence}

Thomas L. Beaumont, Department of Neurological Surgery, Washington University School of Medicine, 660 S. Euclid Ave., Box 8057, St. Louis, MO 63110. email: beaumontt@wustl.edu. 